\title{
Cirugías de mínima invasión
}

Minimally invasive surgeries

Jennifer B. Calzadilla-Orozco ${ }^{a}$, Samanta Hernández-Mayorga ${ }^{b}$

\begin{abstract}
:
At present, it is common for a surgical intervention to suggest several methods for the treatment of conditions, among them, the most common and recommended are minimally invasive surgeries, with the aim of a more comfortable intervention and a more viable recovery for the patient. Since ancient times, methods have been sought so as not to intervene aggressively in procedures that could be so simple without the treatment causing even more damage than the condition itself. The idea arises from the concern to observe the interior of the organism through the natural orifices, this from the era of Hippocrates, the idea was reflected and polished over the years, with the contribution of notable medical figures, in addition to the accelerated technological advance, to become one of the most recommended and successful procedures in the medical area that we know today. The objective of this document is to make the reader aware of all the basic concepts that must be addressed around minimally invasive surgeries, from their concept to their classification.
\end{abstract}

Keywords:

Minimally invasive surgeries, comfort, recovery, security.

\section{Resumen:}

En la actualidad es común que en una intervención quirúrgica se sugieran varios métodos para el tratamiento de las afecciones, entre ellos, las más comunes y recomendadas son las cirugías de mínima invasión, con el fin de una intervención más cómoda y una recuperación más viable para el paciente. Desde la antigüedad se han buscado métodos para no intervenir de manera agresiva en procedimientos que pudieran ser tan simples sin que el tratamiento cause aún más daño que el mismo padecimiento. La idea surge desde la inquietud de observar el interior del organismo por los orificios naturales, esto desde la era de Hipócrates, la idea fue plasmada y pulida con el paso de los años, con el aporte de figuras médicas destacables, además del acelerado avance tecnológico, hasta logarse como uno de los procedimientos más recomendados y exitosos del área médica que conocemos hoy en día. En el presente documento se tiene como objetivo darle a conocer al lector sobre todos aquellos conceptos básicos que se tienen que abordar en torno a las cirugías de mínima invasión, desde su concepto hasta su clasificación.

Palabras Clave:

Cirugías de mínima invasión, comodidad, recuperación, seguridad.

\section{Síntesis}

La cirugía de mínima invasión es una técnica quirúrgica innovadora que produce menos daños en el cuerpo que una cirugía abierta, además de que requiere de pocos recursos para su realización y que presenta grandes beneficios al paciente a corto y largo plazo. El presente documento aborda algunas características de la cirugía de mínima invasión y plantea algunos tipos de éstas.

\footnotetext{
Autor de Correspondencia, Universidad Autónoma del Estado de Hidalgo, https://orcid.org/0000-0003-1404-1500, Email: ca379044@uaeh.edu.mx

b Universidad Autónoma del Estado de Hidalgo, https://orcid.org/0000-0002-8903-6514, Email: he439293@uaeh.edu.mx
} 


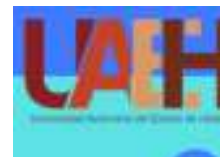

\section{CIRUGÍAS DE} MÍNIMA INVASIÓN

Tepeil del Río

¿Qué son?

Método para realizar operaciones mayores a través de incisiones pequeñas para reducir el troumatismo de la exposición quirúrgica.

Describe con una precisión mayor las incisiones pequeñas que suelen ser necesarias para lograr el acceso a sitios quirúrgicos.
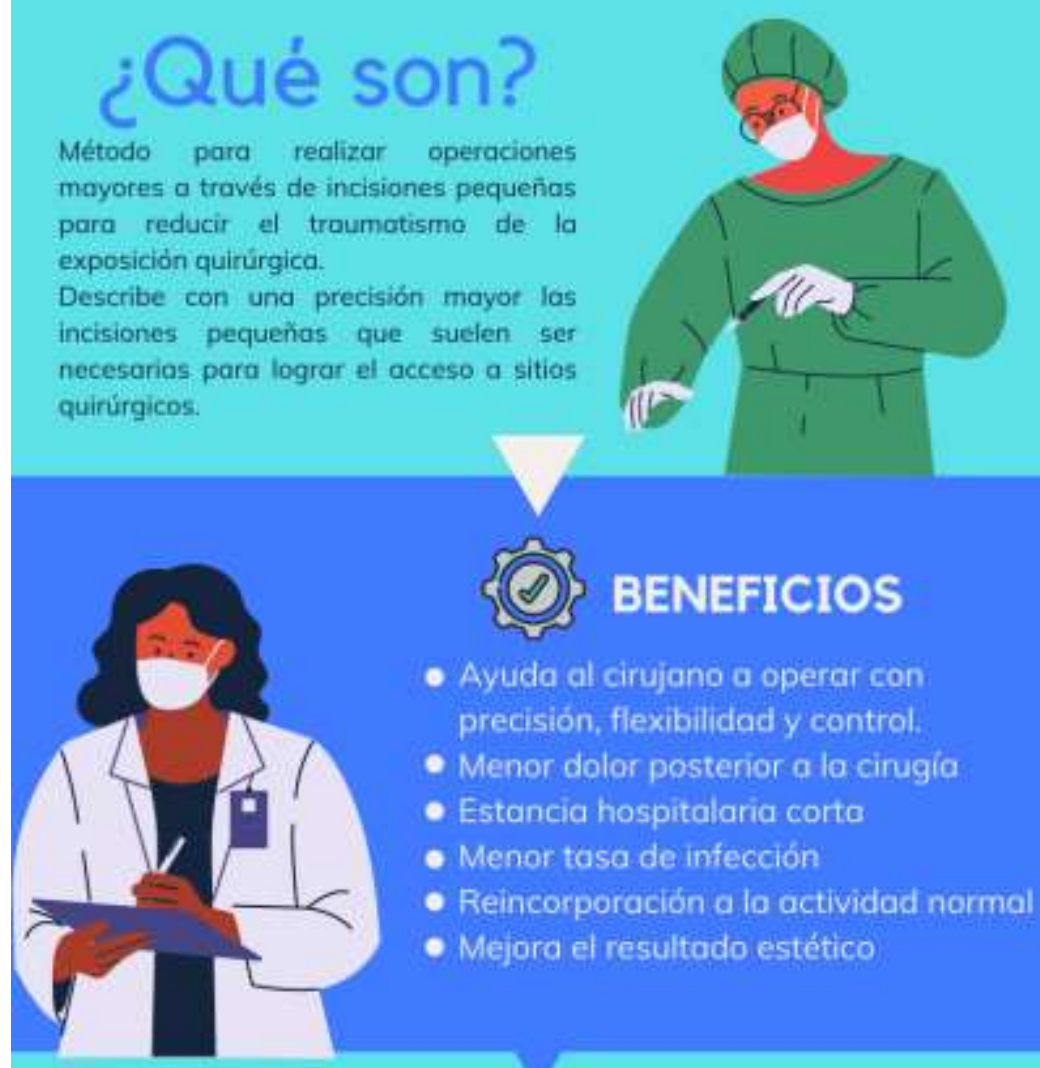

- Ayuda al cirujano a operar con precisión, flexibilidad y control.

- Menor dolor posterior a la cinugía

- Estancia hospitalaria corto

- Menor tasa de infección

- Reincorporación a la actividad narmal

- Mejora el resultado estético

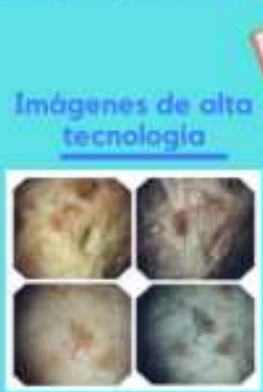

Reducen e!

troumatismo de fo exposición
quirurgica

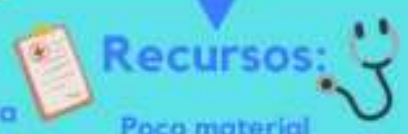

Poco material quirürgico

Uso de pocas

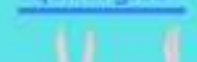
suturos

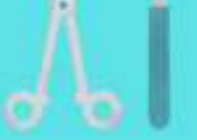

Reduce el costo de la cirugía

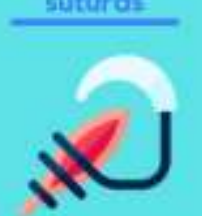

Beneficia para toner una bueno cicatrizoción

\section{TIPOS}

- Adrenalectomía

- Cirugir antirreflujo.

- Cirugía del cáncer

- Colectomía

- Colecistectomic
- Neurocirugía

- Erugía gastroenterologiec

- cirugía cardíaca

- Nefrectomía

- cirugío ortopedica

- Esiblenectomía

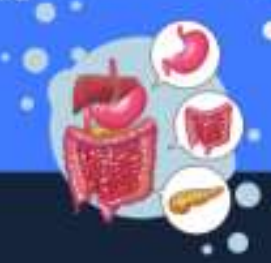




\section{Referencias}

[1] Donn H. Spight \& John G. Hunter (2018). Cirugía de mínima invasión. Recuperado de: https://accessmedicina.mhmedical.com/Content.aspx?bookid $=1513 \&$ sectionid=98624914\#: : text=La\%20cirug\%C3\%ADa\%2 Ode\%20m\%C3\%ADnima\%20invasi\%C3\%B3n\%20es\%20el\%20m \%C3\%A9todo\%20para\%20realizar,traumatismo\%20de\%20la\% 20exposici\%C3\%B3n\%20quir\%C3\%BArgica.

[2] Correa M. (2018) Característica de las cirugías de mínima invasión. Recuperado de: https://www.medicasur.com.mx/es/ms/Cirugia_de_Minima_I nvasion\#: :text=La\%20cirug\%C3\%ADa\%20de\%20m\%C3\%ADni ma\%20invasi\%C3\%B3n,cual\%20se\%20consigue\%20aumentar $\% 20 l a$

[3] Mayo Clinic (2019) Cirugías de invasión mínima. Recuperado de: $\quad$ https://www.mayoclinic.org/es-es/testsprocedures/minimally-invasive-

surgery/about/pac20384771\#: :text=En\%20una\%20cirug\%C3 \%ADa\%20m\%C3\%ADnimamente\%20invasiva,el\%20hospital\%2 0y\%20menos\%20complicaciones. 